\title{
The impact of glutamine deprivation on the expression of MEIS3, SPAG4, LHX1, LHX2, and LHX6 genes in ERN1 knockdown U87 glioma cells
}

\author{
Dariia A. Krasnytska ${ }^{1}$, Olena O. Khita ${ }^{1}$, Dariia O. Tsymbal ${ }^{1}$, Olha Y. Luzina ${ }^{1}$, \\ Anastasiia A. Cherednychenko ${ }^{1}$, Halyna E. KozynkeviCH ${ }^{2}$, Borys H. Bezrodny ${ }^{2}$, \\ Dmytro O. MinchenKo ${ }^{1,2}$
}

${ }^{1}$ Department of Molecular Biology, Palladin Institute of Biochemistry, National Academy of Sciences of Ukraine, Kyiv, Ukraine; ${ }^{2}$ Department of Pediatrics and Department of Surgery, National Bohomolets Medical University, Kyiv, Ukraine E-mail:ominchenko@yahoo.com

Objective. The aim of the current study was to investigate the expression of genes encoded homeobox proteins such as MEIS3 (Meis homeobox 3), SPAG4 (sperm associated antigen 4), LHX1 (LIM homeobox 1), LHX2, and LHX6 in U87 glioma cells in response to glutamine deprivation in control glioma cells and cells with knockdown of ERN1 (endoplasmic reticulum to nucleus signaling 1), the major pathway of the endoplasmic reticulum stress signaling, for evaluation of a possible dependence on the expression of these important regulatory genes from glutamine supply and ERN1 signaling.

Methods. The expression level of MEIS3, SPAG4, LHX, LHX2, and LHX6 genes was studied by real-time quantitative polymerase chain reaction in control U87 glioma cells (transfected by vector) and cells with ERN1 knockdown after exposure to glutamine deprivation.

Results. It was shown that the expression level of MEIS3 and LHX1 genes was up-regulated in control glioma cells treated by glutamine deprivation. At the same time, the expression level of three other genes (LHX2, LHX6, and SPAG4) was down-regulated. Furthermore, ERN1 knockdown significantly modified the effect of glutamine deprivation on $L H X 1$ gene expression in glioma cells, but did not change significantly the sensitivity of all other genes expression to this experimental condition.

Conclusion. The results of this investigation demonstrate that the exposure of U87 glioma cells under glutamine deprivation significantly affected the expression of all genes studied encoding the homeobox proteins and that this effect of glutamine deprivation was independent of the endoplasmic reticulum stress signaling mediated by ERN1, except $L H X 1$ gene.

Key words: U87 glioma cells, ERN1 knockdown, glutamine deprivation, homeobox genes, mRNA expression

Glutamine, as a substrate for glycolysis, is an important for the development and a more aggressive behavior of gliomas, which are highly aggressive tumors with very poor prognosis (Colombo et al. 2011; Yalcin et al. 2014; Zhao et al. 2017; Alimohammadi et al. 2020). Glutamine supply and endoplasmic reticulum stress are very important and complementary factors for the tumor growth and inhibition of ERN1/IRE1 (endoplasmic reticulum to nucleus signaling 1 /inositol requiring enzyme 1) mediated stress signaling can significantly modify the effects of glutamine deprivation on some gene expressions (Minchenko et al. 2013, 2015a, 2021; Tsymbal et al. 2016). It has been shown that ERN1 - XBP1 pathway is also essential for the glucose response and protection of $\beta$ cells (Riabovol et al. 2019). Furthermore,

Corresponding author: Dmytro O. Minchenko, MD, PhD, Department of Molecular Biology, Palladin Institute of Biochemistry, National Academy of Sciences of Ukraine, Leontovycha 9, Kyiv 01030, Ukraine; e-mail: ominchenko@yahoo.com. 
bioinformatics profiling identifies a glucose-related risk signature for the malignancy of glioma (Zhao et al. 2017). At the same time, the complete molecular mechanisms of the interaction of ERN1 mediated stress signaling pathway with glutamine as well as glucose supply are complex yet and warrant further study. Furthermore, there are also data that nutrient starvation is very important factor of cancer cells chemoresistance (Awale et al. 2006). Therefore, arctigenin, a natural lignan compound extracted from Arctium lappa, inhibits the proliferation of various cancer cells and induces tumor cell death under nutrient starvation possibly by inhibiting the unfolded protein response (Awale et al. 2006; Kim et al. 2010; He et al. 2018).

Transcription factors play an important role in malignant tumor growth preferentially through ERN1 (endoplasmic reticulum to nucleus signaling 1) signaling pathway of endoplasmic reticulum stress (Minchenko et al. 2015b, 2015c; Tsymbal et al. 2016). The homeobox proteins represent an important group of transcription factors, which are related to tumorigenesis and are largely investigated (Guca et al. 2018; Le Magnen et al. 2018; Miyashita et al. 2018; Chen et al. 2019; Hamaidi et al. 2019; Li et al. 2019). Recently, it was shown that transcriptional inhibitor ZEB2 (zinc finger E-box binding homeobox 2) functions as an oncogene in human laryngeal squamous cell carcinoma (Li et al. 2019). Furthermore, transcription factor ETS1 (ETS proto-oncogene 1) is coexpressed with ZEB2 and mediates ZEB2-induced epithelial-mesenchymal transition in human tumors (Yalim-Camci et al. 2019).

SPAG4 (sperm associated antigen 4) has a fundamental pathological function in the migration of lung carcinoma cells (Knaup et al. 2014; Ji et al. 2018). Recently, it was shown that SPAG4 mRNA is overexpressed in lung squamous cell carcinoma tissue compared with their paired para-cancerous histological normal tissue samples (Wang et al. 2021). Moreover, SPAG4 could serve as an independent prognostic factor for glioblastoma patients and might be valuable for the clinical management of these patients (Zhang et al. 2021). There are data indicating that MEIS proteins are associated with tumorigenesis, metastasis, and invasion in cancer and may act as tumor suppressors or oncogenes in different cellular settings and that the expression of MEIS3 is upregulated in glioblastoma and many other cancers (Girgin et al. 2020). Furthermore, it was also shown that microRNA-202-3p has tumor-suppressive role in the hepatocellular carcinoma through the KDM3A/ HOXA1/MEIS3 pathway because upregulation of this miRNA reduced the growth of human hepatocellular carcinoma cells in vivo (Zhang et al. 2020).

LIM homeobox proteins (LHX1, LHX2 and LHX6) have also relation to tumor growth (Chen et al. 2019; Hamaidi et al. 2019; Liang et al. 2019; Miyashita et al. 2018; Nathalia et al. 2018). There are data that LHX1 is involved in the expression of various proteins regulating cell movements and EMT in renal carcinoma cells and that silencing of LHX1 decreased pulmonary metastasis in the in vivo model (Hamaidi et al. 2019). Furthermore, LHX1 is an important transcription factor for the histone deacetylase 8 (HDAC8) gene and may play a role in neurogenesis (Ghiaghi et al. 2019). Recently, it was shown that LHX2 acts as a transcriptional activator and is involved in the control of cell differentiation in developing lymphoid and neural cell types and that miR-506 inhibits tumor growth and metastasis via inhibition of WNT/beta-catenin signaling by downregulating LHX2 (Liang et al. 2019). Furthermore, LHX2 promotes malignancy and inhibits autophagy via mTOR in osteosarcoma and is negatively regulated by miR-129-5p (Song et al. 2019). Furthermore, there are also data indicating that LHX2 is capable of proliferation blocking in human $\mathrm{T}$ cell acute lymphoblastic leukemia-derived cells (Miyashita et al. 2018). LHX6 contains the LIM domain, a unique cysteine-rich zinc-binding domain. The encoded protein has tandem LIM domains as well as a DNAbinding homeodomain. Recently, it was shown that transcription factor LHX6 is tumor suppressor and its expression is regulated by DNA methylation and can inhibit the proliferation, invasion and migration through WNT/beta-catenin and TP53 signaling pathways during the Microcystin-LR-induced hepatocarcinogenesis (Chen et al. 2019). Furthermore, miR-1290 promotes proliferation, migration, and invasion of glioma cells by inhibiting LHX6, while LHX6 overexpression inhibited tumor growth (Yan et al. 2018). There are also data indicating that downregulation of miR-214 reverses erlotinib resistance in non-small-cell lung cancer through up-regulation of LHX6 expression (Liao et al. 2017). It is possible that these transcription factors are regulating by long noncoding RNAs in human cancers (Liu et al. 2018).

Malignant tumors use endoplasmic reticulum stress response and its signaling pathways to adapt and enhance tumor cells proliferation under stressful environmental conditions (Almanza et al. 2019; Doultsinos et al. 2017; Manié et al. 2014; Obacz et al. 2017; Papaioannou et al. 2018). It is well known that activation of ERN1 branch of the endoplasmic reticulum stress response is tightly linked to 
apoptosis and cell death and suppression of its function has been demonstrated to result in a significant anti-proliferative effect in glioma growth (Auf et al. 2010; Minchenko et al. 2014; Hetz et al. 2019). Furthermore, inhibition of ERN1 (endoplasmic reticulum to nucleus signaling 1) endoribonuclease has more strong anti-proliferative effect on glioma cells and leads to specific changes in the expression of genes related to ERN1 signaling pathway, the major pathway of the endoplasmic reticulum stress signaling (Auf et al. 2013; Minchenko et al. 2015c).

The aim of the current study was to investigate the expression of genes encoded MEIS3, SPAG4, LHX1, LHX2, and LHX6 homeobox proteins in U87 glioma cells in response to glutamine deprivation in control glioma cells and cells with knockdown of ERN1 for evaluation of possible dependence of the expression of these important regulatory genes from glutamine supply and ERN1 mediated endoplasmic reticulum stress signaling.

\section{Materials and methods}

Cell Lines and Culture Conditions. The glioma cell line U87 was obtained from ATCC (USA) and grown in high glucose (4,5 g/l) Dulbecco's modified Eagle's minimum essential medium (Gibco, Invitrogen, Carlsbad, CA, USA) supplemented with glutamine (2 mM), 10\% fetal bovine serum (Equitech-Bio, Inc., USA), penicillin (100 units/ml; Gibco) and streptomycin $\left(0.1 \mathrm{mg} / \mathrm{ml}\right.$; Gibco) at $37^{\circ} \mathrm{C}$ in incubator with $5 \% \mathrm{CO}_{2}$. In this work we used two sublines of these cells. One subline was obtained by selection of stable transfected clones with overexpression of vector pcDNA3.1, which was used for creation of dnERN1. These untreated glioma cells were used as control
1 (control glioma cells) in the study of the effect of glutamine deprivation on the level of gene expressions. Second subline was obtained by selection of stable transfected clones with overexpression of ERN1 dominant/negative construct (dnERN1), having suppression of both the protein kinase and endoribonuclease activities of this signaling enzyme (Auf et al. 2010). It has been shown that cells with dnERN1 have a lower proliferation rate, do not express spliced variant of XBP1, a key transcription factor in ERN1 signaling, and have not the phosphorylated isoform of ERN1 after induction of endoplasmic reticulum stress by tunicamycin (Auf et al. 2010, 2013; Minchenko et al. 2015c). The impact of glutamine deprivation on the expression of MEIS3, SPAG4, LHX, LHX2, and LHX6 genes in the cells with a deficiency of ERN1, introduced by dnERN1, was compared with cells transfected with the previously mentioned, empty vector pcDNA3.1 (control glioma cells). Both used in this study sublines of glioma cells are grown in the presence of geneticin (G418) while these cells carrying empty vector pcDNA3.1 or dnERN1 construct. Glutamine deprivation condition were created by changing the complete DMEM medium into culture plates on DMEM medium without glutamine and plates were exposed to this condition for $16 \mathrm{hrs}$.

RNA isolation. Total RNA was extracted from glioma cells using the Trizol reagent according to manufacturer's protocol (Invitrogen, Carlsbad, CA, USA). The RNA pellets was washed with $75 \%$ ethanol and dissolved in nuclease-free water. For additional purification RNA samples were re-precipitated with 95\% ethanol and re-dissolved again in nuclease-free water. RNA concentration and spectral characteristics were measured using NanoDrop Spectrophotometer ND1000 (PEQLAB, Biotechnologie GmbH).

Table 1

Characteristics of the primers used for quantitative real-time polymerase chain reaction

\begin{tabular}{|c|c|c|c|c|}
\hline Gene symbol & Gene name & Primer's sequence & $\begin{array}{c}\text { Nucleotide numbers in } \\
\text { sequence }\end{array}$ & $\begin{array}{c}\text { GenBank accession } \\
\text { number }\end{array}$ \\
\hline MEIS3 & Meis homeobox 3 & $\begin{array}{l}\text { F: 5'-cagcctcccagaccagaata } \\
\text { R: 5'-tcctggtccaagtcctcatc }\end{array}$ & $\begin{array}{l}1028-1047 \\
1222-1203\end{array}$ & NM_020160.3 \\
\hline SPAG4 & sperm associated antigen 4 & $\begin{array}{l}\text { F: 5'-cagcttctggaactacgcac } \\
\text { R: 5'-gtggatgctgcagagtgatg }\end{array}$ & $\begin{array}{l}1042-1061 \\
1193-1174\end{array}$ & NM_003116.3 \\
\hline LHX1 & LIM homeobox 1 & $\begin{array}{l}\text { F: 5'-atttatttccgttcccgccg } \\
\text { R: 5'-ccaaaactcgcaccaggaaa }\end{array}$ & $\begin{array}{l}354-373 \\
591-572\end{array}$ & NM_005568.5 \\
\hline LHX2 & LIM homeobox 2 & $\begin{array}{l}\text { F: } 5^{\prime} \text {-tccctactacaatggcgtgg } \\
\text { R: } 5^{\prime} \text {-gtcggggttgtggttaatgg }\end{array}$ & $\begin{array}{l}1214-1233 \\
1457-1438\end{array}$ & NM_004789.4 \\
\hline LHX6 & LIM homeobox 6 & $\begin{array}{l}\text { F: 5'-cggaacagctgcaggttatg } \\
\text { R: 5'-ctgaacggggtgtagtggat }\end{array}$ & $\begin{array}{c}987-1006 \\
1224-1205\end{array}$ & NM_014368.5 \\
\hline ACTB & beta-actin & $\begin{array}{l}\text { F: } 5 \text { '-ggacttcgagcaagagatgg } \\
\text { R: } 5 \text {-agcactgtgttggcgtacag }\end{array}$ & $\begin{array}{l}747-766 \\
980-961\end{array}$ & NM_001101 \\
\hline
\end{tabular}


Reverse transcription and quantitative PCR analysis. Thermo Scientific Verso cDNA Synthesis Kit (Germany) was used for reverse transcription as described (Minchenko et al. 2018). The expression levels of homeobox mRNAs as well as ACTB mRNA were measured in control U87 glioma cells and cells with a deficiency of ERN1, introduced by dnERN1, by quantitative polymerase chain reaction using SYBRGreen Mix (ABgene, Thermo Fisher Scientific, Epsom, Surrey, UK) and "QuantStudio 5 Real-Time PCR System” (Applied Biosystems, USA). Polymerase chain reaction was performed in triplicate. The expression of beta-actin mRNA was used as control of analyzed RNA quantity. The pair of primers specific for each studied gene was received from Sigma-Aldrich (St. Louis, MO, U.S.A.) and Metabion Int. AG (Martinstried, Germany) and used for quantitative polymerase chain reaction (Table 1).

Quantitative PCR analysis. This analysis was performed using a special computer program "Differential expression calculator" and statistical analysis using Excel program and OriginPro 7.5 software as described previously (Minchenko et al. 2015c).

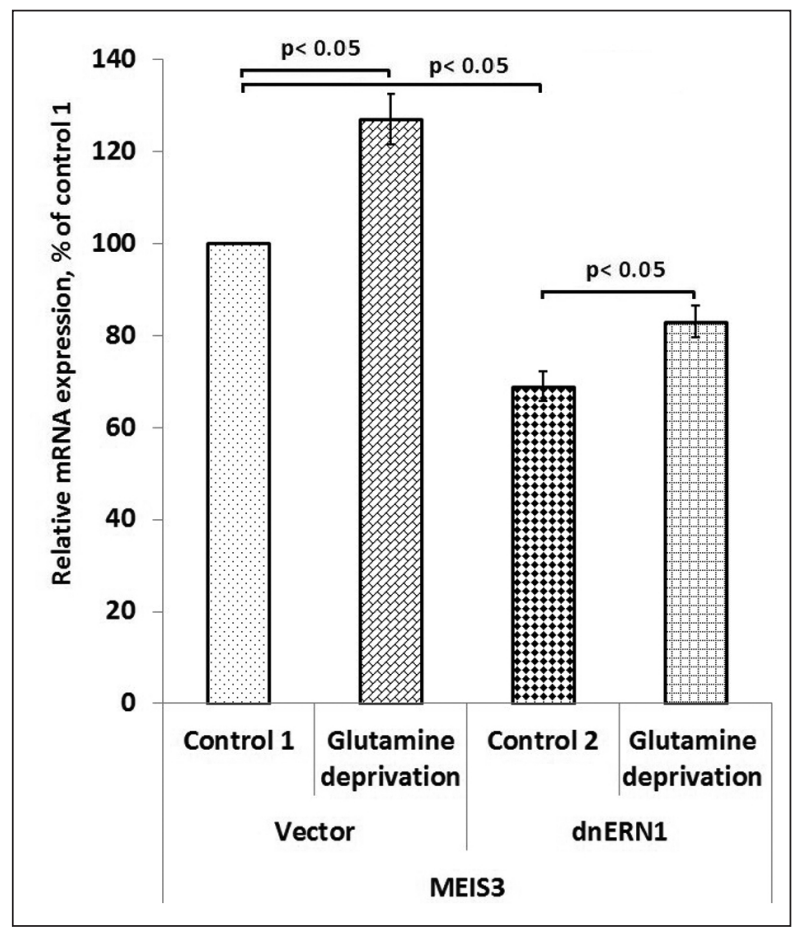

Figure 1. Effect of glutamine deprivation on the expression level of Meis homeobox 3 (MEIS3) mRNA in control U87 glioma cells (Vector) and cells with a blockade of the ERN1 signaling protein by dnERN1 measured by qPCR. Values of MEIS3 mRNA expression were normalized to beta-actin mRNA level and represented as percent for control $1(100 \%) ; n=4$.
Comparison of two means was performed by the use of two-tailed Student's $t$-test. A $\mathrm{p}<0.05$ was considered significant in all cases. The values of studied gene expressions were normalized to the expression of beta-actin mRNA and represent as percent of control $(100 \%)$. All values are expressed as mean \pm SEM from triplicate measurements performed in 4 independent experiments. The amplified DNA fragments were also analyzed on a $2 \%$ agarose gel and that visualized by SYBR ${ }^{\star}$ Safe DNA Gel Stain (Life Technologies, Carlsbad, CA, USA).

\section{Results}

To reveal a possible role of glutamine deprivation and endoplasmic reticulum stress signaling mediated by ERN1 protein in the expression level of homeobox genes in U87 glioma cells, we studied the effect of glutamine deprivation on MEIS3, SPAG4, LHX, LHX2, and LHX6 gene expressions in cells with and without ERN1 functional activity. As shown in Figure 1, the expression of Meis homeobox 3 (MEIS3) mRNA in control glioma cells, transfected

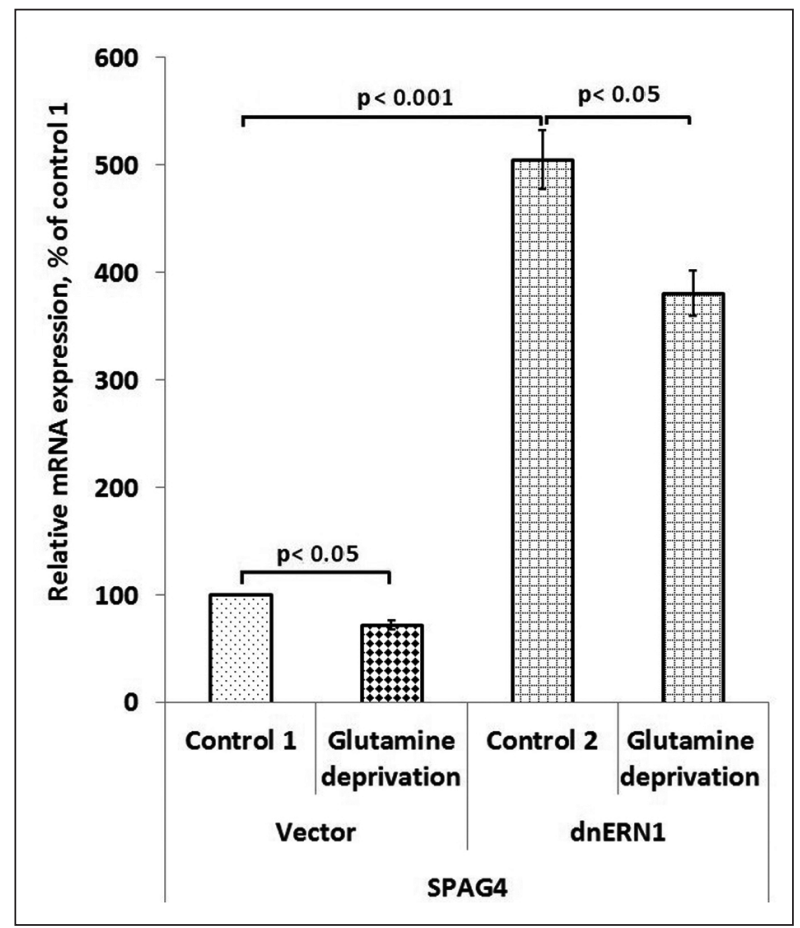

Figure 2. Effect of glutamine deprivation on the expression level of sperm associated antigen 4 (SPAG4) mRNA in control U87 glioma cells (Vector) and cells with a blockade of the ERN1 by dnERN1 measured by qPCR. Values of SPAG4 mRNA expression were normalized to beta-actin mRNA level and represented as percent for control 1 (100\%); NS - no significant changes; $\mathrm{n}=4$. 


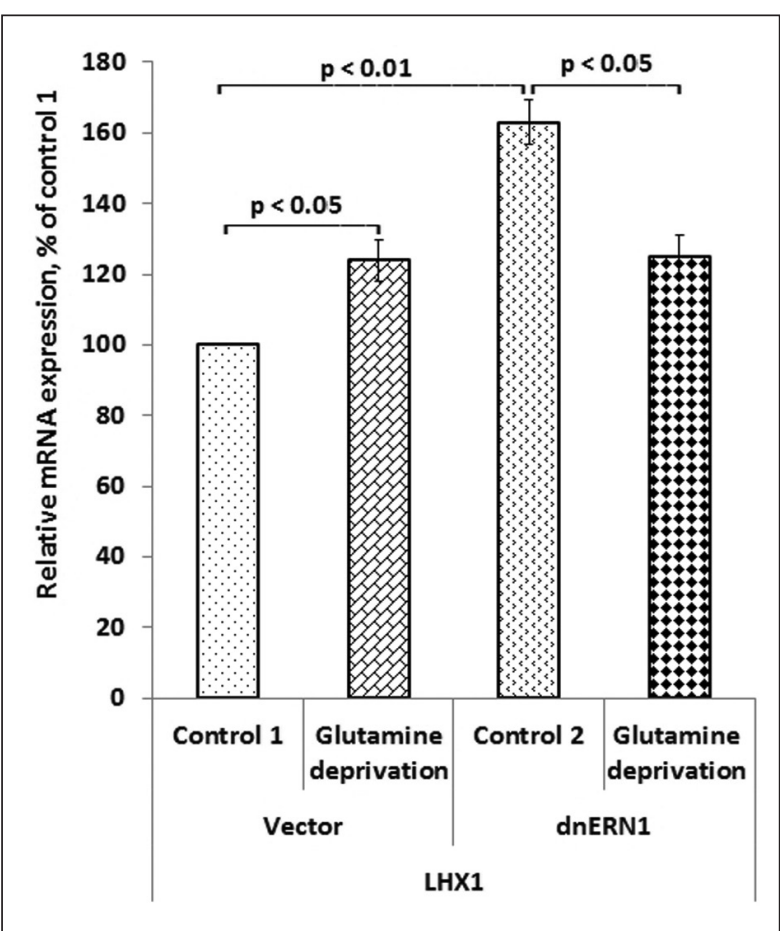

Figure 3. Effect of glutamine deprivation on the expression level of LIM homeobox 1 (LHX1) mRNA in control U87 glioma cells (Vector) and cells with a blockade of the ERN1 by dnERN1 measured by qPCR. Values of LHX1 mRNA expression were normalized to beta-actin mRNA level and represented as percent for control $1(100 \%) ; n=4$.

by empty vector pcDNA3.1, increased after exposure to glutamine deprivation ( $+27 \%)$ in comparison with the cells growing in complete DMEM medium. Furthermore, inhibition of ERN1 signaling enzyme function by dnERN1 not significantly modified the sensitivity of MEIS3 gene expression to this experimental condition $(+20 \%)$ in comparison with corresponding control (Figure 1). Next, we investigated the effect of glutamine deprivation on the expression of gene encoding SPAG4 (sperm associated antigen 4) in relation to inhibition of ERN1 function. As shown in Figure 2, glutamine deprivation resulted in a significant down-regulation of this homeobox gene expression $(-28 \%)$ in comparison with control glioma cells, transfected by empty vector. Furthermore, inhibition of ERN1 signaling protein function led to similar changes in the expression level of this gene $(-25 \%$; Figure 2).

We also studied the effect of glutamine deprivation on the expression of gene encoding LIM homeobox 1 (LHX1) protein in glioma cells in relation to complete inhibition of ERN1 signaling enzyme function. It

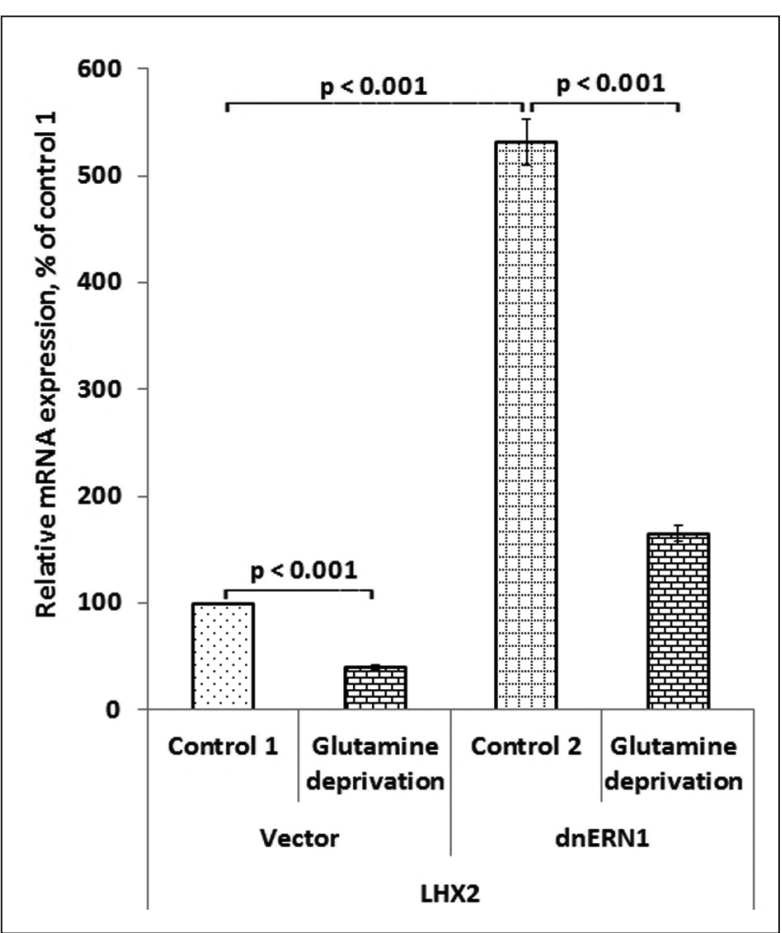

Figure 4. Effect of glutamine deprivation on the expression level of LIM homeobox 2 (LHX2) mRNA in control U87 glioma cells (Vector) and cells with a blockade of the ERN1 by dnERN1 measured by qPCR. Values of LHX2 mRNA expression were normalized to beta-actin mRNA level and represented as percent for control $1(100 \%) ; n=4$.

was shown that the expression level of $L H X 1$ gene is up-regulated $(+24 \%)$ in control glioma cells (transfected by empty vector) under glutamine deprivation in comparison with the cells growing in regular medium (Figure 4). At the same time, inhibition of ERN1 signaling enzyme function significantly decreased the effect of glutamine deprivation on this homeobox gene expression $(-29 \%)$ as compared to corresponding control cells (transfected by dnERN1; Figure 3).

As shown in Figure 4, exposure of control glioma cells to glutamine deprivation led to significant downregulation of LHX2 mRNA expressions (-60\%) in comparison with cells growing with glutamine in the medium. Furthermore, inhibition of both enzymatic activities of ERN1 does not significantly modify the effect of glutamine deprivation on the expression of this homeobox gene expression (-69\%). Furthermore, the expression of LIM homeobox 6 gene was also down-regulated $(-30 \%)$ in control glioma cells treated by glutamine deprivation and inhibition of ERN1 did not significantly change the sensitivity of 
this LIM homeobox gene to glutamine deprivation in comparison with the corresponding control cells growing with glutamine (Figure 5).

Thus, glutamine deprivation affects the expression of different homeobox genes in gene-specific manner and these effects of glutamine deprivation on gene expressions were preferentially resistant to inhibition of ERN1 signaling. Results of this investigation are summarized in Figure 6, which clearly demonstrates the gene-specific character of changes in the expression profile of studied homeobox genes in glioma cells under glutamine deprivation independently from ERN1 inhibition, except $L H X 1$ gene.

\section{Discussion}

In this work, we studied the effect of glutamine deprivation on the expression of genes encoding important homeobox proteins, which control transcription preferentially as transcription factors, in U87 glioma cells in relation to inhibition of ERN1, the major signaling pathway of the unfolded protein response. For this purpose, we used control glioma cells, transfected by empty vector pcDNA3.1, and cells with full ERN1 deficiency introduced by dnERN1. This is important for the evaluation of possible significance of glutamine deprivation in ERN1 dependent control of glioma cell proliferation because there are data indicating that the endoplasmic reticulum stress signaling mediated by ERN1 is involved in numerous metabolic pathways and ERN1 knockdown has clear anti-tumor effect (Auf et al. 2010, 2013; Bravo et al. 2013; Manié et al. 2014; Minchenko et al. 2014, 2015 a, c; Logue et al. 2018). Results of our study clarify possible mechanisms of glutamine deprivation on the proliferation/surviving of ERN1 knockdown glioma cells through specific changes in the expression profile of genes encoding important transcription factors of homeobox family especially LHX1.

We showed that glutamine deprivation leads to significant up-regulation of the expression of MEIS3 gene in both control and ERN1 knockdown glioma cells. Furthermore, these changes in MEIS3 gene expression under glutamine deprivation are essentially independent of ERN1 inhibition. There are data indicating that the expression of this gene is up-regulated in glioblastoma as well as in many other tumors (Girgin et al. 2020). It is possible that the up-regulation of MEIS3 gene under glutamine deprivation reflects compensatory mechanism connected with decreased proliferation rate of glioma cells under nutrient starvation (Awale et al. 2006; Colombo et al. 2011; Drogat et al. 2007; Kim et al. 2010; He et al.

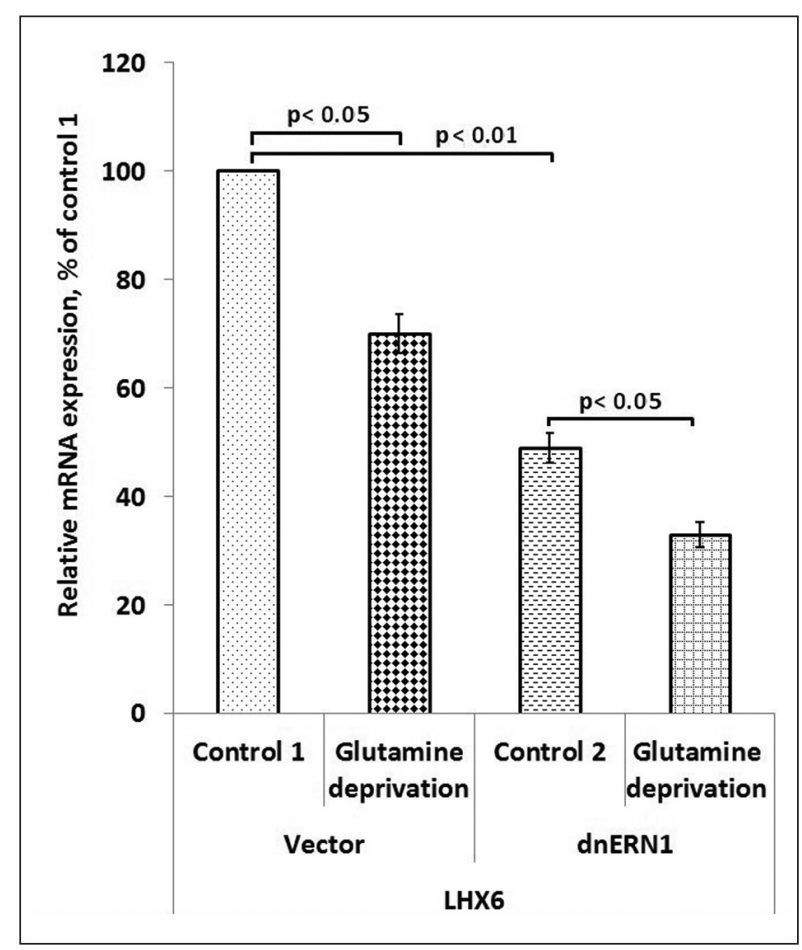

Figure 5. Effect of glutamine deprivation on the expression level of LIM homeobox 6 (LHX6) mRNA in control U87 glioma cells (Vector) and cells with a blockade of the ERN1 by dnERN1 measured by qPCR. Values of LHX6 mRNA expression were normalized to beta-actin mRNA level and represented as percent for control $1(100 \%) ; n=4$.

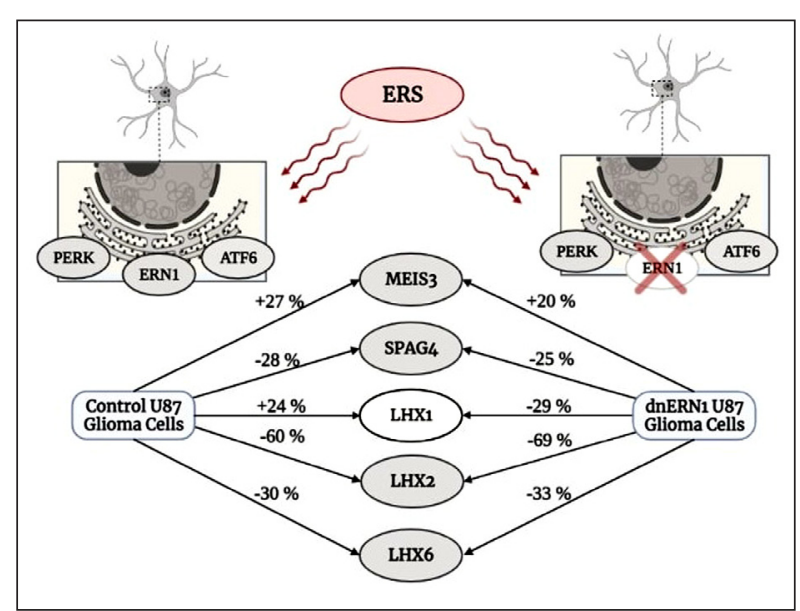

Figure 6. Schematic demonstration of changes in the expression profile of MEIS3, SPAG4, LHX, LHX2, and LHX6 genes in the control and ERN1 knockdown U87 glioma cells under glutamine deprivation.

2018). At the same time, the expression level of SPAG4 gene is down-regulated in control glioma cells under glutamine deprivation. Furthermore, similar changes 
in the expression of this gene we have received in cells with ERN1 knockdown. The protein encoded by SPAG4 gene has a fundamental pathological function in the migration of lung carcinoma cells (Knaup et al., 2014; Ji et al., 2018; Zhang et al. 2020) and downregulation of its expression under glutamine deprivation condition in both control and ERN1 knockdown glioma cells possibly reflects a glutamine and proliferation dependent character of this gene expression because glutamine supply is very important factor for tumor growth (Colombo et al. 2011).

Results of this study also demonstrate variable effects of glutamine deprivation on the expression of LIM homeobox genes encoding proteins LHX1, LHX2, and LHX6, which have diverse effects on tumor growth (Chen et al. 2019; Hamaidi et al., 2019; Liang et al., 2019; Miyashita et al., 2018; Nathalia et al., 2018). Thus, the exposure of control gliomas cells without glutamine leads to the up-regulation of LHX1 gene expression and down-regulation of the expression of LHX2 and LHX6 genes. At the same time, we showed that ERN1 knockdown in glioma cells leads to suppression of $L H X 1$ gene expression under glutamine deprivation and does not significantly modulate the effect of this treatment on the expression of LHX2 and LHX6 genes. Functional significance of ERN1 knockdown mediated changes in the expression of $L N X 1$ gene under glutamine deprivation condition is possibly connected with suppression of ERN1 knockdown glioma cells proliferation (Auf et al. 2010) because this transcription factor functions as pro-proliferative tumor factor (Ghiaghi et al., 2019; Hamaidi et al., 2019). However, the expression of LHX2 and LHX6 genes is reduced under conditions of glutamine deficiency, although these genes have different functional significance
(Liao et al., 2017; Liang et al., 2019; Miyashita et al., 2018; Nathalia et al., 2018). Moreover, the expression of $L H X 2$ and $L H X 6$ genes is resistant to inhibition of ERN1 signaling. It is possible that down-regulation of the expression of LHX2 and LHX6 genes under glutamine deprivation in both control and ERN1 knockdown glioma cells possibly reflects a glutamine dependent as well as proliferation related character of these gene expressions because glutamine is very important for tumor growth (Colombo et al. 2011; Drogat et al. 2007).

In conclusion. This study provides unique insights into the molecular mechanisms regulating the expression of genes encoding some homeobox transcription factors in glioma cells in response to glutamine deprivation and their correlation with inhibition of ERN1 activity and reduced cell proliferation in cells harboring dnERN1, attesting to the fact that endoplasmic reticulum stress as well as glutamine are necessary components of the glioma growth and cell survival. Furthermore, our results validate interaction of endoplasmic reticulum stress signaling pathway ERN1 with glutamine deprivation in gene-specific manner, in particular in the regulation of LHX1 gene expression encoding homeobox transcription factor for the histone deacetylase 8 and many other genes and may play a role in neurogenesis. The detailed molecular mechanisms of this regulation have not been yet clearly defined and warrant further investigation.

\section{Acknowledgement}

This work was funded by the State Budget Program "Support for the Development of Priority Areas of Scientific Research” (Code: 6541230).

\section{References}

Alimohammadi E, Bagheri SR, Salehi AS, Rizevandi P, Rezaie Z, Abdi A. Prognostic factors in patients with glioblastoma multiforme: focus on the pathologic variants. Acta Neurol Belg 120, 1341-1350, 2020.

Almanza A, Carlesso A, Chintha C, Creedican S, Doultsinos D, Leuzzi B, Luis A, McCarthy N, Montibeller L, More S, Papaioannou A, Puschel F, Sassano ML, Skoko J, Agostinis P, de Belleroche J, Eriksson LA, Fulda S, Gorman AM, Healy S, Kozlov A, Muñoz-Pinedo C, Rehm M, Chevet E, Samali A. Endoplasmic reticulum stress signalling - from basic mechanisms to clinical applications. FEBS J 286, 241-278, 2019.

AufG, Jabouille A, Guerit S, Pineau R, Delugin M, Bouchecareilh M, Favereaux A, Maitre M, Gaiser T, von Deimling A, Czabanka M, Vajkoczy P, Chevet E, Bikfalvi A, Moenner M. A shift from an angiogenic to invasive phenotype induced in malignant glioma by inhibition of the unfolded protein response sensor IRE1. Proc Natl Acad Sci USA 107, 15553 -15558, 2010.

Auf G, Jabouille A, Delugin M, Guerit S, Pineau R, North S, Platonova N, Maitre M, Favereaux A, Vajkoczy P, Seno M, Bikfalvi A, Minchenko D, Minchenko O, Moenner M. High epiregulin expression in human U87 glioma cells relies on IRE1alpha and promotes autocrine growth through EGF receptor. BMC Cancer 13, 597, 2013. 
Awale S, Lu J, Kalauni SK, Kurashima Y, Tezuka Y, Kadota S, Esumi H. Identification of arctigenin as an antitumor agent having the ability to eliminate the tolerance of cancer cells to nutrient starvation. Cancer Res 66, 1751-1757, 2006.

Bravo R, Parra V, Gatica D, Rodriguez AE, Torrealba N, Paredes F, Wang ZV, Zorzano A, Hill JA, Jaimovich E, Quest AF, Lavandero S. Endoplasmic reticulum and the unfolded protein response: dynamics and metabolic integration. Int Rev Cell Mol Biol 301, 215-290, 2013.

Chen HQ, Zhao J, Li Y, Huang YJ, Chen DJ, He LX, Wang LQ, Zheng CF, Wang J, Cao J, Shu WQ, Liu JY, Liu WB. Epigenetic inactivation of LHX6 mediated microcystin-LR induced hepatocarcinogenesis via the Wnt/betacatenin and P53 signaling pathways. Environ Pollut 252, 216-226, 2019.

Colombo SL, Palacios-Callender M, Frakich N, Carcamo S, Kovacs I, Tudzarova S, Moncada S. Molecular basis for the differential use of glucose and glutamine in cell proliferation as revealed by synchronized HeLa cells. Proc Natl Acad Sci USA 108, 21069-21074, 2011.

Doultsinos D, Avril T, Lhomond S, Dejeans N, Guédat P, Chevet E. Control of the Unfolded Protein Response in Health and Disease. SLAS Discov 22, 787-800, 2017.

Drogat B, Bouchecareilh M, North S, Petibois C, Deleris G, Chevet E, Bikfalvi A, Moenner M. Acute L-glutamine deprivation compromises VEGF-a upregulation in A549/8 human carcinoma cells. J Cell Physiol 212, 463-472,2007.

Ghiaghi M, Forouzesh F, Rahimi H. Effect of Sodium Butyrate on LHX1 mRNA Expression as a Transcription Factor of HDAC8 in Human Colorectal Cancer Cell Lines. Avicenna J Med Biotechnol 11, 317-324, 2019.

Girgin B, Karadag-Alpaslan M, Kocabas F. Oncogenic and tumor suppressor function of MEIS and associated factors. Turk J Biol 44, 328-355, 2020.

Guca E, Sunol D, Ruiz L, Konkol A, Cordero J, Torner C, Aragon E, Martin-Malpartida P, Riera A, Macias MJ. TGIF1 homeodomain interacts with Smad MH1 domain and represses TGF-beta signaling. Nucleic Acids Res 46, 9220-9235, 2018.

Hamaidi I, Coquard C, Danilin S, Dormoy V, Beraud C, Rothhut S, Barthelmebs M, Benkirane-Jessel N, Lindner V, Lang H, Massfelder T. The Lim1 oncogene as a new therapeutic target for metastatic human renal cell carcinoma. Oncogene 38, 60-72, 2019.

He Y, Fan Q, Cai T, Huang W, Xie X, Wen Y, Shi Z. Molecular Mechanisms of the Action of Arctigenin in Cancer. Biomed Pharmacother 108, 403-407, 2018.

Hetz C, Axten JM, Patterson JB. Pharmacological targeting of the unfolded protein response for disease intervention. Nat Chem Biol 15, 764-775, 2019.

Ji Y, Jiang J, Huang L, Feng W, Zhang Z, Jin L, Xing X. Spermassociated antigen 4 (SPAG4) as a new cancer marker interacts with Nesprin 3 to regulate cell migration in lung carcinoma. Oncol Rep 40, 783-792, 2018.

Kim JY, Hwang JH, Cha MR, Yoon MY, Son ES, Tomida A, Ko B, Song SW, Shin-ya K, Hwang YI, Park HR. Arctigenin Blocks the Unfolded Protein Response and Shows Therapeutic Antitumor Activity. J Cell Physiol 224, 33-40, 2010.

Knaup KX, Monti J, Hackenbeck T, Jobst-Schwan T, Klanke B, Schietke RE, Wacker I, Behrens J, Amann K, Eckardt KU, Warnecke C, Wiesener MS. Hypoxia regulates the sperm associated antigen 4 (SPAG4) via HIF, which is expressed in renal clear cell carcinoma and promotes migration and invasion in vitro. Mol Carcinog 53, 970-978, 2014.

Le Magnen C, Virk RK, Dutta A, Kim JY, Panja S, Lopez-Bujanda ZA, Califano A, Drake CG, Mitrofanova A, AbateShen C. Cooperation of loss of NKX3.1 and inflammation in prostate cancer initiation. Dis Model Mech 11, dmm035139, 2018.

Li Q, Ma L, Wu Z, Wang G, Huang Q, Shen Z, Yu R. Zinc finger Ebox binding homeobox 2 functions as an oncogene in human laryngeal squamous cell carcinoma. Mol Med Rep 19, 4545-4552, 2019.

Liang TS, Zheng YJ, Wang J, Zhao JY, Yang DK, Liu ZS. MicroRNA-506 inhibits tumor growth and metastasis in nasopharyngeal carcinoma through the inactivation of the Wnt/beta-catenin signaling pathway by downregulating LHX2. J Exp Clin Cancer Res 38, 97, 2019.

Liao J, Lin J, Lin D, Zou C, Kurata J, Lin R, He Z, SuY. Down-regulation of miR-214 reverses erlotinib resistance in non-small-cell lung cancer through up-regulating LHX6 expression. Sci Rep 7, 781, 2017.

Liu Z, Dai J, Shen H. Systematic analysis reveals long noncoding RNAs regulating neighboring transcription factors in human cancers. Biochim Biophys Acta Mol Basis Dis 1864, 2785-2792, 2018.

Logue SE, McGrath EP, Cleary P, Greene S, Mnich K, Almanza A, Chevet E, Dwyer RM, Oommen A, Legembre P, Godey F, Madden EC, Leuzzi B, Obacz J, Zeng Q, Patterson JB, Jäger R, Gorman AM, Samali A. Inhibition of IRE1 RNase activity modulates the tumor cell secretome and enhances response to chemotherapy. Nat Commun 9, 3267, 2018. 
Manié SN, Lebeau J, Chevet E. Cellular mechanisms of endoplasmic reticulum stress signaling in health and disease. 3. Orchestrating the unfolded protein response in oncogenesis: an update. Am J Physiol Cell Physiol 307, C901-C907, 2014.

Minchenko DO, Kharkova AP, Hubenia OV, Minchenko OH. Insulin receptor, IRS1, IRS2, INSIG1, INSIG2, RRAD, and BAIAP2 gene expressions in glioma U87 cells with ERN1 loss of function: effect of hypoxia and glutamine or glucose deprivation. Endocr Reg 47, 15-26, 2013.

Minchenko DO, Danilovskyi SV, Kryvdiuk IV, Bakalets TV, Lypova NM, Karbovskyi LL, Minchenko OH. Inhibition of ERN1 modifies the hypoxic regulation of the expression of TP53-related genes in U87 glioma cells. Endoplasm Reticul Stress Dis 1, 18-26, 2014.

Minchenko DO, Kharkova AP, Tsymbal DO, Karbovskyi LL, Minchenko OH. IRE1 inhibition affects the expression of insulin-like growth factor binding protein genes and modifies its sensitivity to glucose deprivation in U87 glioma cells. Endocr Reg 49, 185-197, 2015a.

Minchenko OH, Tsymbal DO, Minchenko DO, Kovalevska OV, Karbovskyi LL, Bikfalvi A. Inhibition of ERN1 signaling enzyme affects hypoxic regulation of the expression of E2F8, EPAS1, HOXC6, ATF3, TBX3 and FOXF1 genes in U87 glioma cells. Ukr Biochem J 87(2): 76-87, $2015 \mathrm{~b}$.

Minchenko OH, Tsymbal DO, Moenner M, Kovalevska OV, Lypova NM. Inhibition of the endoribonuclease of ERN1 signaling enzyme affects the expression of proliferation-related genes in U87 glioma cells. Endoplasm Reticul Stress Dis 2, 18-29, 2015c.

Minchenko OH, Tsymbal DO, Minchenko DO, Hnatiuk OS, Prylutskyy YI, Prylutska SV, Tsierkezos NG, Ritter U. Single-walled carbon nanotubes affect the expression of genes associated with immune response in normal human astrocytes. Toxicology in vitro 52, 122-130, 2018.

Minchenko DO, Khita OO, Tsymbal DO, Viletska YM, Sliusar MY, Yefimova YV, Levadna LO, Krasnytska DA, Minchenko OH. ERN1 knockdown modifies the impact of glucose and glutamine deprivations on the expression of EDN1 and its receptors in glioma cells. Endocr Reg 55, 72-82, 2021.

Miyashita K, Kitajima K, Goyama S, Kitamura T, Hara T. Overexpression of Lhx2 suppresses proliferation of human T cell acute lymphoblastic leukemia-derived cells, partly by reducing LMO2 protein levels. Biochem Biophys Res Commun 495, 2310-2316, 2018.

Nathalia E, Theardy MS, Elvira S, Rosellinny G, Liyanto AS, Utama MP, Sumarpo A. Downregulation of tumorsuppressor gene LHX6 in cancer: a systematic review. Rom J Intern Med 56, 135-142, 2018.

Obacz J, Avril T, Le Reste PJ, Urra H, Quillien V, Hetz C, Chevet E. Endoplasmic reticulum proteostasis in glioblastoma-From molecular mechanisms to therapeutic perspectives. Sci Signal 10(470), pii: eaal2323, 2017.

Papaioannou A, Chevet E. Driving Cancer Tumorigenesis and Metastasis Through UPR Signaling. Curr Top Microbiol Immunol 414, 159-192, 2018.

Riabovol OO, Tsymbal DO, Minchenko DO, Lebid-Biletska KM, Sliusar MY, Rudnytska OV, Minchenko OH. Effect of glucose deprivation on the expression of genes encoding glucocorticoid receptor and some related factors in ERN1-knockdown U87 glioma cells. Endocr Regul 53, 237-249, 2019.

Song H, Liu J, Wu X, Zhou Y, Chen X, Chen J, Deng K, Mao C, Huang S, Liu Z. LHX2 promotes malignancy and inhibits autophagy via mTOR in osteosarcoma and is negatively regulated by miR-129-5p. Aging (Albany NY) 11, 9794-9810, 2019.

Tsymbal DO, Minchenko DO, Kryvdiuk IV, Riabovol OO, Halkin OV, Ratushna OO, Minchenko OH. Expression of proliferation related transcription factor genes in U87 glioma cells with IRE1 knockdown upon glucose and glutamine deprivation. Fiziol Zh 62, 3-15, 2016.

Wang Y, Tang Y, Li J, Wang D, Li W. Human sperm-associated antigen 4 as a potential prognostic biomarker of lung squamous cell carcinoma. J Int Med Res 49, 03000605211032807, 2021.

Yalcin A, Clem BF, Imbert-Fernandez Y, Ozcan SC, Peker S, O’Neal J, Klarer AC, Clem AL, Telang S, Chesney J. 6-Phosphofructo-2-kinase (PFKFB3) promotes cell cycle progression and suppresses apoptosis via Cdk1mediated phosphorylation of p27. Cell Death and Disease 5, e1337, 2014.

Yalim-Camci I, Balcik-Ercin P, Cetin M, Odabas G, Tokay N, Sayan AE, Yagci T. ETS1 is coexpressed with ZEB2 and mediates ZEB2-induced epithelial-mesenchymal transition in human tumors. Mol Carcinog 58, 1068-1081, 2019.

Yan L, Cai K, Sun K, Gui J, Liang J. MiR-1290 promotes proliferation, migration, and invasion of glioma cells by targeting LHX6. J Cell Physiol 233, 6621-6629, 2018.

Zhang C, Wang M, Ji F, Peng Y, Wang B, Zhao J, Wu J, Zhao H. A Novel Glucose Metabolism-Related Gene Signature for Overall Survival Prediction in Patients with Glioblastoma. Biomed Res Int 2021, 8872977, 2021. 
Zhang Y, Pan Q, Shao Z. Tumor-Suppressive Role of microRNA-202-3p in Hepatocellular Carcinoma Through the KDM3A/HOXA1/MEIS3 Pathway. Front Cell Dev Biol 8, 556004, 2020.

Zhao S, Cai J, Li J, Bao G, Li D, Li Y, Zhai X, Jiang C, Fan L. Bioinformatic Profiling Identifies a Glucose-Related Risk Signature for the Malignancy of Glioma and the Survival of Patients. Mol Neurobiol 54, 8203-8210, 2017. 